\title{
Neuropatía compresiva del nervio interoseo posterior a nivel del codo (síndrome de la arcada de frohse) ¿debe incluirse en el listado de enfermedades profesionales?
}

\author{
Must the neuropathy compressive of posterior interoseal nerve at the elbow level \\ (arcade of frohse syndrome) be included in the occupational diseases list?
}

\section{Raúl Jesús Regal Ramos}

Unidad Médica del Equipo de Valoración de Incapacidades del Instituto

Nacional de la Seguridad Social de la Provincial de Madrid. Madrid. España.

Recibido: 31-05-10

Aceptado: $31-08-10$

\section{Correspondencia:}

Raúl Jesús Regal Ramos

Canillas, $22-3^{\circ} \mathrm{B}$

28002 Madrid. España.

Tfno: 626193169

e-mail: raul-jesus.regal@inss.seg-social.es o raulregal@hotmail.com

Resumen

Título: Neuropatía Compresiva del Nervio Interoseo Posterior a nivel del codo (Síndrome de la Arcada de Frohse) ¿debe incluirse en el Listado de Enfermedades Profesionales?

Introducción: La afectación compresiva del nervio radial se encuentra recogida en el último Listado de Enfermedades Profesionales (LEP), con el código 2F0601. En este apartado no se recoge entre las "principales actividades" capaces de producir afectación del n.radial la compresión de origen laboral más frecuente de este, el síndrome de la Arcada de Frohse (SAF).

Objetivos: El objetivo de esta revisión no es solo reflejar que la afectación compresiva del nervio interóseo posterior puede considerarse una Enfermedad Profesional (EP), sino que además debería estar recogida en el actual listado de EP entre las "principales actividades capaces de producir afectación del nervio radial".

Metodología: Se han revisado hasta Febrero de 2010 las siguientes bases de datos bibliográficas: Medline, Embase, Cochrane.

Resultados: Esta revisión bibliográfica nos permite concluir que: El SAF puede tener un origen laboral (la fibrosis del supinador corto se relaciona con movimientos repetidos de pronación y supinación del antebrazo y esta descrita su mayor prevalencia en determinadas profesiones que realizan estos movimientos). El SAF es la neuropatía compresiva de origen laboral mas frecuente del nervio radial, la más relacionada con los movimientos repetitivos de la mano y antebrazo. La Arcada de Frohse es el lugar más frecuente de compresión del radial.

Conclusiones: Por tanto, el SAF puede considerarse una EP, si asocia factores de riesgo laborales suficientes, y debería estar recogido en el LEP por tratarse de la localización más frecuente de compresión de origen laboral del nervio radial.

Med Segur Trab (Internet) 2010; XX (220): 248-256

Palabras clave: Nervio radial. Arcada de Frohse. Nervio interoseo posterior. Enfermedades profesionales. 


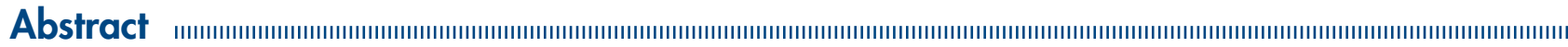

Title: Must the Neuropathy Compressive of Posterior Interoseal Nerve at the elbow level (Arcade of Frohse syndrome) be included in the Occupational Diseases List?

Introduction: The compress affection of radial nerve is included in the last Occupational Diseases List (ODL), with code 2f0601. In this paragraph the Arcade of Frohse syndrome (SAF) isn't included among the "main activities" able to induce radial nerve affection, the occupational origin radial nerve compression more frequent.

Objectives: The objective of this review isn't only to show that the compress affection of posterior interosseous nerve can be considered a occupational diseases, but, also, it should be included in the current ODL, among the "main activities able to induce radial nerve affection".

Material and methods: To February 2010 it have been reviewed the following bibliographical database: Medline, Embase, Cochrane.

Results: This bibliographical review allow us to conclude: SAF can have a occupational origin (fibrosis of short supinator is related with forearm repetitive pronosupinacion movements, and it is more prevalent in certain professions in which are made these kind of movements). SAF is the occupational origin neuropathy compress more frequent of radial nerve, and the most related with hand and forearm repetitive movements. The Arcade of Frohse is the most common radial compression place.

Conclusions: Therefore, SAF can be considered a occupational diseases if it carries enough occupational risks factors, and it should be included in the ODL, by being the most frequent occupational origin compression of radial nerve.

Med Segur Trab (Internet) 2010; XX (220): 248-256

Key words: Radial nerve. Arcade of Fröhse. Posterior interosseous nerve. Occupational Diseases. 


\section{INTRODUCCIÓN}

Las neuropatías compresivas son la patología neurológica periférica más frecuente del miembro superior y son una causa frecuente de pérdida de días laborales en las empresas $^{1,2}$. Entre las distintas etiologías de dicha compresión tenemos fracturas, luxaciones, sinovitis, gangliones, tumores y el origen laboral

Las neuropatías compresivas de origen laboral se definen ${ }^{3}$ como aquella lesiones nerviosas producidas por traumatismos repetidos a los nervios periféricos como consecuencia de las tareas desempeñadas en el puesto de trabajo que implican posturas forzadas mantenidas, esfuerzos o movimientos repetidos $\mathrm{y}$ apoyos prolongados $\mathrm{o}$ mantenidos. La compresión reiterada del nervio daña los vasos epineurales causando un edema que puede llevar a una fibrosis y esta al deterioro progresivo del nervio ${ }^{4}$.

Las dos causas más frecuentes de neuropatía compresiva en miembro superior son ${ }^{5}$ el síndrome del túnel del carpo (la de mayor prevalencia en el mundo laboral) y la compresión del nervio cubital particularmente susceptible en el codo (túnel cubital) y en la muñeca (canal de Guyon)

La incidencia exacta del atrapamiento del nervio interoseo posterior (PIN), rama motora del radial, no está aclarada, de cualquier manera, un estudio epidemiológico sobre neuropatías compresiva demostró una incidencia de neuropatía compresiva radial de 2,97 por 100.000 en hombres y de 1,42 por 100.000 en mujeres ${ }^{5}$. Arle JE et al ${ }^{6}$ sostienen que el síndrome de atrapamiento del nervio radial idiopático representa aproximadamente un 0 '7\% de las lesiones no traumáticas de la extremidad superior, sin embargo otros autores como Erak $\mathrm{S}$ y Smola $\mathrm{C}^{7,8}$ sostienen que se trata, en cuanto a frecuencia, de la segunda neuropatía por compresión tras el síndrome del túnel carpiano, por la frecuente asociación de la compresión del PIN con epicondilitis.

Son muy infrecuentes las afectaciónes del nervio circunflejo, supraescapular y musculocutáneo

La afectación compresiva del nervio radial se encuentra recogida en el último listado de Enfermedades Profesionales (LEP), RD 1299/2006 ${ }^{9}$, con el código 2F0601 y en este apartado se recoge entre las "principales actividades" capaces de producir afectación del n. radial : "Trabajos que causan un apoyo prolongado sobre las tabaqueras anatómicas", "Movimientos extremos de hiperflexión e hipertensión" y "Trabajos que entrañen contracción repetida del músculo supinador largo, como conductores de automóviles, presión crónica por uso de tijeras". Las relaciones de actividades recogidas en este listado, aunque en muchas ocasiones son muy detalladas, nunca deben de entenderse como relaciones cerradas, pues el propio encabezamiento habla de "principales actividades capaces de producir enfermedades relacionadas con el agente". Este hecho hace que la patología a la que nos referimos en esta revisión pueda ser considerada una Enfermedad Profesional (EP) pese a no venir literalmente recogida en el RD 1299/2006. No obstante, dado que la literatura científica reconoce a la compresión del PIN como la neuropatía compresiva de origen laboral más frecuente del nervio radial, consideramos que si debería estar recogida esta patología, o sus mecanismos, causales en el actual LEP

\section{OBJETIVOS}

El objetivo de esta revisión no es solo reflejar que la afectación compresiva del nervio interóseo posterior puede considerarse una EP, sino que además debería estar recogida en el actual listado de EP entre las "principales actividades capaces de producir afectación del nervio radial"

\section{METODOLOGÍA}

Se han revisado hasta Febrero de 2010 las siguientes bases de datos bibliográficas: Medline, Embase, Cochrane. 


\section{RECUERDO ANATÓMICO}

La compresión del radial puede producirse a distintos niveles ${ }^{10}$

A nivel de la axila se han descrito parálisis radiales producidas por el uso de muletas o por herramientas que comprimen la axila, en fundidores y estampadores de metal. Pueden producirse también por apoyos prolongados del brazo sobre superficies duras, frecuentes durante el sueño profundo en mala posición (parálisis de los sábados)

Antes de llegar al codo, el nervio radial rodea la diafisis humeral desde dentro hacia fuera y luego desciende en el canal bicipital externo (Figura 1). En la cara anterior del codo encontramos un espacio anatómico definido como "túnel o desfiladero radial", que comienza y está limitado posteriormente por el cóndilo y termina en la porción distal del músculo supinador corto ${ }^{11}$. El "túnel radial" consta a su vez de 3 porciones ${ }^{12}$. La primera porción se extiende desde el sitio en que el nervio radial perfora el tabique intermuscular lateral del brazo hasta el nivel de la interlínea de la articulación húmero-radial del codo. La segunda porción se extiende desde la interlínea articular del codo hasta el borde superior del haz superficial del músculo supinador corto. Este borde recibe el nombre de "Arcada de Frohse". Es en esta porción donde el nervio radial, entre 1,5 y 2,5 cm. por debajo de la interlínea articular del codo, se divide en sus dos ramos terminales: rama superficial (anterior y sensitiva) o n. radial sensitivo superficial, que desciende el compartimento anterior del antebrazo por debajo del supinador largo y por fuera de la arteria radial superficial; y rama posterior (profundo y motor) o $\mathrm{n}$. interóseo posterior, que se introduce bajo en la Arcada de Frohse (Figura 2). Finalmente, la tercera porción se extiende desde la AF hasta el borde inferior del músculo supinador.

Figura 1

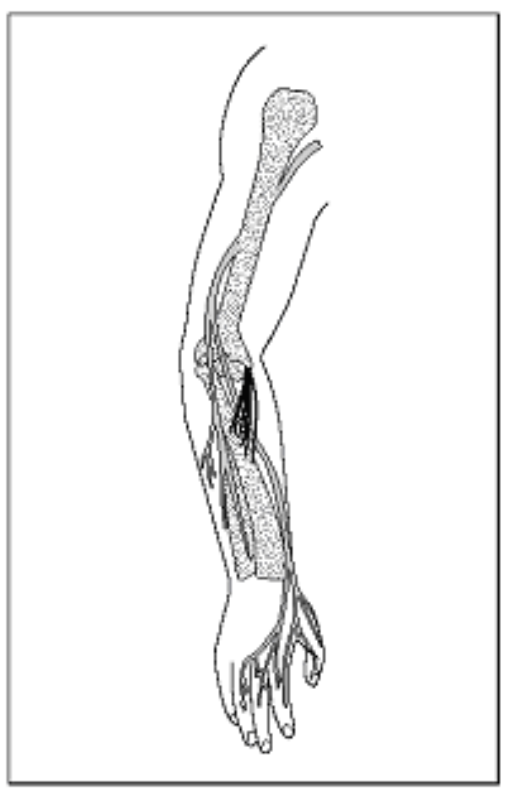

A nivel de la muñeca puede producirse la compresión de la rama sensitiva superficial del nervio radial, dando lugar al Síndrome de Wartenberg. El síndrome de Wartenberg es un cuadro clínico descrito por el autor del mismo nombre en 1932 como "queiralgia parestésica" ${ }^{13}$ y que presenta clínicamente dolor en la superficie radial dorsal del antebrazo con irradiación al dorso de la mano, el pulgar y el segundo y tercer dedos. La denominó así por la similitud de la meralgia parestésica, por compresión del nervio cutáneo lateral en el muslo. La etiología de este síndrome se ha asociado, a multitud de cuadros, entre ellos la neuropatía compresiva, siendo la más frecuente a nivel del músculo supinador largo, en el momento en que el nervio se hace dorsal bordeando el tendón del citado músculo, es decir, en la zona radial del antebrazo o de la muñeca (parálisis de los esposados $)^{3}$ 
Por último, podemos observar la lesión de la rama digital dorsal del nervio radial. Aparece por el uso de herramientas, como tijeras, cuya empuñadura apoya sobre el territorio radial del pulgar. El cuadro clínico, consistente en parestesias y disestesias en el lado radial de la falange distal del pulgar ${ }^{3}$.

De todos los cuadros anteriormente descritos, la afectación del PIN destaca por ser la neuropatía compresiva del nervio radial más relacionada con los movimientos repetitivos de la mano y antebrazo ${ }^{4-26}$ y la Arcada de Frohse se ha descrito como el lugar más frecuente de compresión del PIN $^{19,24,27,28}$ y como el lugar más frecuente de compresión del nervio radial ${ }^{2,23,27,29-36 . ~}$

\section{FISIOPATOLOGÍA}

El nervio radial a su paso por el túnel radial puede comprimirse por distintas causas $^{25}$ :

- las bandas fibrosas anteriores de la cabeza radial

- la arteria radial recurrente que puede comprimir el nervio contra la cabeza del radio (cuerda de Henry)

- el origen tendinoso del segundo radial externo (extensor carpi radialis brevis) que puede improntar el nervio en la pronación completa

- la arcada de Frohse, la más frecuente ${ }^{28,37}$.

- el borde distal de salida del supinador.

- El músculo supinador corto (M. Supinator) tiene una parte superficial y otra profunda y entre ambas se desliza el PIN siguiendo un trayecto espiroideo inverso hasta que alcanza la cara posterior del antebrazo

El borde proximal del fascículo superficial del músculo supinador corto se denomina Arcada de Frohse ${ }^{38}$. Este borde muscular puede sufrir cambios histológicos que conducirían a su transformación en una banda fibrosa, cambios histológicos que estarían relacionados con los movimientos repetidos del codo, especialmente movimientos de pronosupinación (22-23). Cuando este borde se fibrosa en un grado suficiente se produce una compresión del PIN, dando lugar al Síndrome de la Arcada de Frohse.

\section{CLÍNICA}

El atrapamiento del PIN puede presentarse de dos formas diferentes y con una nomenclatura diferente en la literatura:

1. Síndrome del Nervio Interóseo Posterior: Parálisis no dolorosa, en ocasiones precedida por un cuadro de dolor en la cara extensora del antebrazo

2. Síndrome del Túnel Radial (STR): Más que debilidad o parálisis los pacientes con STR presentan dolor en la cara lateral del antebrazo proximal, cuadro doloroso muy difícil de diferenciar de la epicondilitis lateral o "codo de tenista" por lo que se ha denominado "codo de tenista resistente". El PIN al ser nervio motor puro no produce alteraciones de la sensibilidad, pero si puede causar dolor local ${ }^{39}$.

Los términos Síndrome de la Arcada de Frohse o Síndrome de la Celda del Supinador, engloba ambas presentaciones clínicas

La diferencia en la presentación clínica puede atribuirse a una diferencia en el grado de compresión del nervio pero al comprimirse el mismo nervio, el tratamiento de ambas presentaciones se puede abordar con las mismas intervenciones quirúrgicas ${ }^{40}$. 


\section{RELACIÓN LABORAL}

Diversos estudios apoyan la hipótesis de que los movimientos repetidos de pronación y supinación causan compresión del nervio radial a nivel del Túnel Radial ${ }^{14-26}$.

En este sentido los trabajadores con ocupaciones que requieren tareas manuales repetitivas tienen particularmente riesgo de desarrollar este síndrome ${ }^{21,41}$.

Esta patología tiene especial incidencia en violinistas ${ }^{42-44}$, directores de orquesta (15-16,45), trabajadores manuales que realizan movimientos repetidos de atornillar y desatornillar ${ }^{19}$ y tenistas ${ }^{46,47}$, entre otros ${ }^{48}$. Pero el Síndrome de la Arcada de Frohse se ha descrito en múltiples profesiones como camareros ${ }^{49}$, fabricadores de corsés, lecheros ${ }^{50} \mathrm{e}$ incluso en nadadores ${ }^{51}$.

\section{RELACIÓN CON EL ACTUAL LISTADO DE ENFERMEDADES PROFESIONALES}

Como hemos comentado al principio de este artículo la afectación compresiva del nervio radial se encuentra recogida en el LEP con el código 2F0601 y en este apartado se recogen las siguientes actividades: "Trabajos que causan un apoyo prolongado sobre las tabaqueras anatómicas", "Movimientos extremos de hiperflexión e hipertensión”, "Trabajos que entrañen contracción repetida del músculo supinador largo, como conductores de automóviles, presión crónica por uso de tijeras"

Como hemos visto el "apoyo prolongado sobre las tabaqueras anatómicas" no es causa de atrapamiento del nervio interóseo posterior, aunque se ha descrito un caso en un marine del ejército americano después de portar durante un periodo largo de tiempo una ametralladora $\mathrm{M} 60^{52}$.

Los movimientos extremos de hiperflexión e hiperextensión, no son movimientos que realice en músculo supinador corto. El músculo supinador corto y el bíceps, son los 2 únicos que producen supinación del antebrazo y mientras que el bíceps es supinador y flexor, el supinador corto es solo supinador (si el antebrazo está en extensión, solo este músculo es capaz de producir la supinación del antebrazo) ${ }^{53}$.

La contracción repetida del músculo supinador largo tampoco es causa de atrapamiento del nervio interóseo posterior. Cuando hablamos de músculo supinador largo nos estamos refiriendo al músculo brachio-radialis, y cuando hablamos de músculo supinador corto al músculo supinator. Las funciones del músculo brachio-radialis son principalmente la flexión y supinación del codo. Este músculo se relaciona anatómicamente con el nervio radial sensitivo superficial pero no con el PIN (Figura 2). 


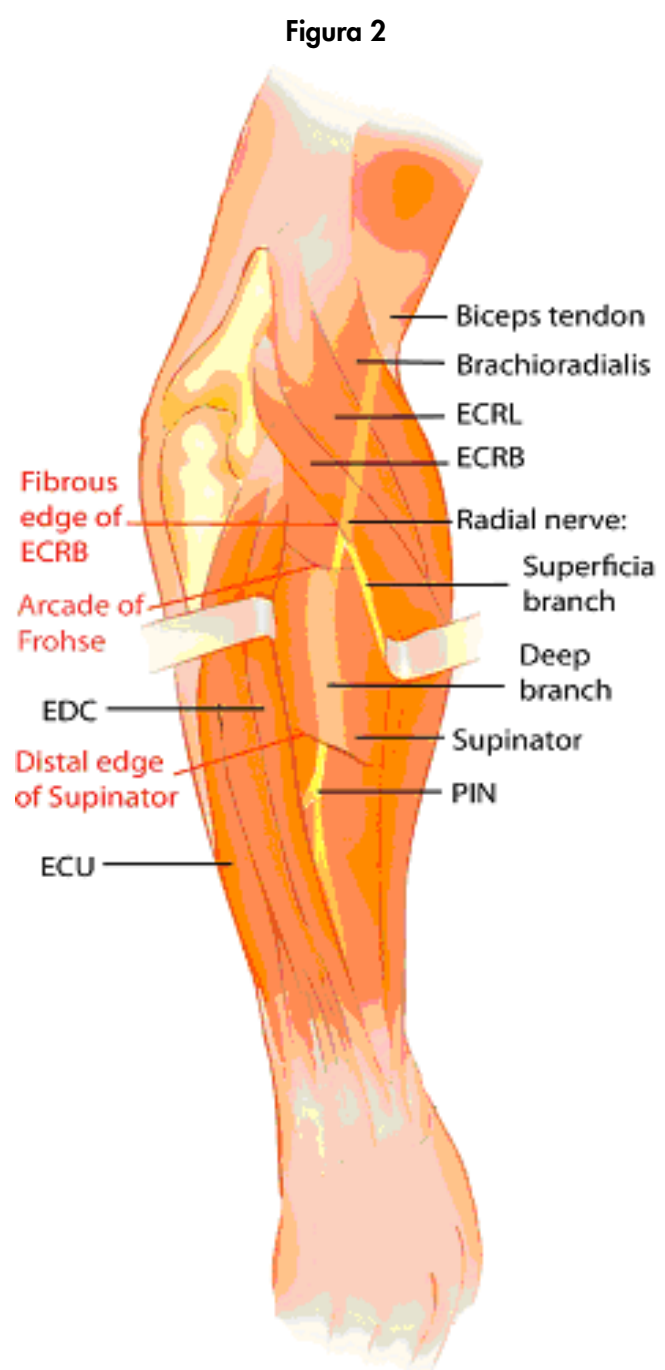

Por último la presión crónica por uso de tijeras, causaría la lesión de la rama digital dorsal del nervio radial

Aunque la relación de actividades recogidas nunca debe entenderse cerrada, lo cierto es que el Síndrome de la Arcada de Frohse pese a ser la compresión de origen laboral más frecuente del nervio radial, no viene recogido en el LEP.

\section{CONCLUSIONES}

El Síndrome de la Arcada de Froshe es una neuropatía compresiva que afecta a la rama motora del nervio radial, PIN, y que se debe al atrapamiento del nervio dentro de un músculo supinador corto anormalmente endurecido o fibrosado en su parte proximal.

Esta revisión bibliográfica nos permite concluir que:

- El Síndrome de la Arcada de Froshe puede tener un origen laboral. La fibrosis del supinador corto se relaciona con movimientos repetidos de pronación y supinación del antebrazo y esta descrita su mayor prevalencia en determinadas profesiones que realizan estos movimientos ${ }^{15,19,21,41-47}$.

- El Síndrome de la Arcada de Froshe es la neuropatía compresiva de origen laboral mas frecuente del nervio radial, la más relacionada con los movimientos repetitivos de la mano y antebrazo ${ }^{14-26}$.

— La Arcada de Frohse es el lugar más frecuente de compresión del radial ${ }^{12,23,27,29-36}$. 
Por tanto, el Síndrome de la Arcada de Frohse puede considerarse una EP, si asocia factores de riesgo laborales suficientes, y debería estar recogido en el Listado de EP por tratarse de la localización más frecuente de compresión de origen laboral del nervio radial

\section{BIBLIOGRAFIA}

1. Fisher DL, Andres RO, Airth D, Smith SS. Repetitive motion disorders: the design of optimal rate-rest profiles. Hum Factors 1993;35(2):283-304

2. Himmelstein JS et al. Work-related upper-extremity disorders and work disability: clinical and psychosocial presentation. J Occup Environ Med 1995;37(11):1278-86

3. Consejo Interterritorial del Sistema Nacional de Salud. «Protocolo de vigilancia sanitaria específica para los/ as trabajadores/as expuestos a neuropatías por presión"; 2.000

4. Rydevik B, Lundborg G. Permeability of intraneural microvessels and perineurium following acute, graded experimental nerve compression. Scand J Plast Reconstr Surg 1977;11:179 -187

5. Latinovic R, Gulliford MC, Hughes RA. Incidence of common compressive neuropathies in primary care. J Neurol Neurosurg Psychiatry 2006; 77:263-265.

6. Arle J, Zager E. Surgical treatment of common entrapment neuropathies in the upper limbs. Muscle Nerve $2000 ; 23: 1160-74$

7. Erak S, Day R, Wang A. The role of supinator in the pathogenesis of chronic lateral elbow pain: a biomechanical study

8. Smola C. About the problem of radial tunnel syndrome or "where does tennis elbow end and where does the radial tunnel syndrome begin? Hand Chir Mikrochir 2004 Aug;36(4):241-5.

9. Real Decreto $1299 / 2006$ de 10 de noviembre, por el que se aprueba el cuadro de Enfermedades Profesionales

10. Plate AM, Green SM. Compressive radial neuropathies. Instr Course Lect 2000;49:295-304.

11. Barnum M, Mastey RD, Weiss AP, Akelman E. Radial tunnel syndrome.Hand Clin 1996; 12:4, 679-689

12. Bertone Vicente Hugo, Ottone Nicolás Ernesto, Lo Tártaro Maximiliano Ariel, García de Quirós Nicolás, Arrotea Agustín, Domínguez Mario et al. Consideraciones Anátomo-Clínicas sobre el Nervio Radial en el Codo. Int. J. Morphol. [revista en la Internet]. 2008 Jun; 26(2): 437-444. Disponible en: http://www.scielo. $\mathrm{cl} /$ scielo.php?script $=\quad$ sci_arttext\&pid=S0717-95022008000200032\&lng=es. $\quad$ doi: $10.4067 / \mathrm{S} 0717-$ 95022008000200032

13. Wartenberg R. Cheiralgia Paraesthetic. Isolierte neuritis desramus superficialis nervi radialis. Z Ges Neurol Psychiatr 1935;141:145-155

14. Kopell G, Thompson W. Peripheral entrapment neuropathies. Baltimore: William and Wilkins; 1963

15. Guillain G, Courtellemont G. L'action du muscle court supinateur dans la paralyse du nerf radial. Pathogénie d'une paralyse radiale incomplete cher une chef d'orcheste".Press.Med. 1905, 7: 50-52

16. Comtet JJ, Chambaud D. Paralyse spontanée du nerf inter-osseux posterieur pur lesion inhabituelle. Deux observations. Rev.Chir.Orthop. 1975,61:533-541

17. Luppnino T. Considerazioni anatomo-cliniche sune alcune varie sindromi nervosa canalicolari dll“arto superiore. Riv.Chir.Mano 1972, 10: 74-82

18. Bonnel F: Nerf radial. Bonnel F, Mansat M. Nerfs périphériques. Ed. Masson, Paris, 1990, 8-89.

19. Rus Mompo, Antonio. Arcada de Frohse .Rev. Esp. de Cir. Ost 1989 :101-108

20. Serrano M.F , Gomez, A. Disorders of the hand for accumulative trauma in the work Rev Iberoam Fisioter Kinesiol. 2004;7:41-61

21. Roquelaure Y, Raimbeau G, Dano C, Martin YH, Pelier Cady MC, Mechali S, et al. Occupational risk factors for radial tunnel syndrome in industrial workers. Scand J Work Environ Health 2000; 26(6):507-13,

22. Clavert P, Lutz JC, Adam P, Wolfram-Gabel R, Liverneaux P, Kahn JL. Frohse's arcade is not the exclusive compression site of the radial nerve in its tunnel.Rev Chir Orthop Traumatol. 2009 Apr;95(2):114-8,

23. Spinner, M. The arcade of Frohse and its relationship to posterior interosseus nerve paralisys. J.Bone Joint Sur. 1968,50 B: 809-812

24. Cravens G, Kline D. Posterior interosseous nerve palsies. Neurosurgery 1990;27, 397-302 
25. Konjengbam M, Elangbam J. Radial nerve in the radial tunnel: anatomic sites of entrapment. Clin Anat 2004;17(1):21-25

26. Wilhwam A. Philadelphia: WB. Saunders; 1993

27. Ozturk A, Kutlu C, Taskara N, Kale A, Bayraktar B, Cecen A. Anatomic and morphometric study of the arcade of Frohse in cadavers. Sur Radiol Anat 2005;27:171- 5

28. Boyer MI, Hastings H 2nd. Lateral tennis elbow: "Is there any science out there?" J Shoulder Elbow Surg 1999;8(5):481-491.

29. Gonzalez R. E. La arcada de Frohse y sus relaciones neurovasculares. XXI Congreso de la Sociedad Rioplatense de Anatomía, 1984.,

30. Lafalla, R. Relaciones de la rama posterior del nervio radial con la cabeza del radio e inervación motora de los músculos posteriores del antebrazo. XVII Congreso de la Sociedad Rioplatense de Anatomía, 1980

31. Debouck C, Rooze M. The arcade of Frohse: an anatomical study. Surg Radiol Anat 1995;17:245-8.

32. Kirici Y, Irmak M. Investigation of two possible compression sites of the deep branch of the radial nerve and nerve supply of the extensor carpi radialis brevis muscle. Neurol. Med. Chir. (Tokyo), 44:14-9, 2004

33. Salvá G, Terrados X, Perec C. Neuropatía compresiva del nervio radial en el codo: Diagnóstico y tratamiento. Medicina Balear 2008; 23-26

34. Roust R, Espeche R. C. División del nervio radial en el pliegue del codo. V Congreso de la Sociedad Rioplatense de Anatomía. Mendoza, 1969.

35. De Santolo A. La mano dormida por compresiones nerviosas. Gac Méd Caracas, dic. 2005, vol.113, no.4, p.485-499. ISSN 0367-4762

36. Lowe J, Sen S, Mackinnon S. Current Approach to Radial Nerve Paralysis. Plastic and Reconstructive Surgery 15 September 2002: 110: 1099-1113

37. Prasartritha T, Luipolvanish P, Rojanakit A. A study of the posterior interosseous nerve (PIN) and the radial tunnel in 30 Thai cadavers. J Hand Surg. 1993;18:107-12

38. Frohse, F. y Frankel,M. Die Muskeln des menschlichen Armes. Jena.G. Fisher Verlag 1908

39. Gutiérrez E. Diagnóstico neurofisiológico de enfermedades neurológicas producidas por movimientos repetidos. Tercera reunión de Grupo de Estudio de Neurología del Trabajo de la SEN. Barcelona, 2000

40. Alan C. Dang, MD, Craig M. Rodner, MD . Unusual Compression Neuropathies of the Forearm, Part I: Radial Nerve. JHS 2009Dec. Vol 34 A,1906-14

41. Lawrence $\mathrm{T}$, Mobbs $\mathrm{P}$, Fortems $\mathrm{Y}$, Stanley JK. Radial tunnel syndrome. A retrospective review of 30 decompressions of the radial nerve. J Hand Surg [Br] 1995;20(4):454-9.

42. Terrono AL, Millender LH. Management of work-related upper-extremity nerve entrapments. Orthop Clin North Am 1996;27(4):783-93

43. Charness ME, Parry Gc, Markinson RE. Entrapment neuropathies in musicians. Neurology 1985;35:40-5

44. Maffulli N, Maffulli F. Transiet entrapment neuropathy of the posterior interosseous nerve in violin players. J NeurosurgPsychiatry 1991;54:65-7.

45. Orozco L, Solé J. Tecnopatías del Músico. Prevención y tratamiento de las lesiones y enfermedades profesionales de instrumentistas y cantantes. Introducción a la medicina de la danza. Aritza Comunicación S.I

46. Gabel GT, Morrey BF. Tennis elbow. Instr Course Lect. 1998;47:165-72

47. Pfandl S, Wetzel R, Hackspacher J, Puhl W. Supinator tunnel syndrome, a differential diagnosis of socalled tennis elbow.. Sportverletz Sportschaden 1992;6(2):71-76

48. Dang AC, Rodner CM. Unusual compression neuropathies of the forearm, part I: radial nerve. J Hand Surg Am. 2009 Dec;34 (10):1906-14

49. Hobhouse N, Heald C. A case of posterior interosseous paralysis.BMJ 1936;1: 841

50. Weinberger L. Non-traumatic paralysis of the dorsal interosseous nerve. Surg Gynecol Obstet 1939;69:358

51. Kruse F. Paralysis of the dorsal interosseous nerve not due to direct trauma: a case showing spontaneous recovery. Neurology 1958;8: 307.

52. Sonna LA, Scott BR. Posterior interosseous nerve palsy in a machina gunner. Mil Med 1995;160:364 -366

53. Orts Llorca, F. Anatomía Humana. Científico-Médica. Ged. 1986

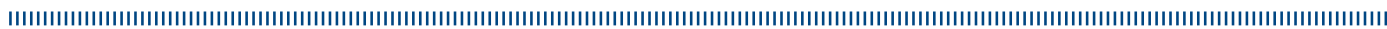

